\title{
Erratum to: Popliteus impingement after TKA may occur with well-sized prostheses
}

\author{
Michel P. Bonnin ${ }^{1,2} \cdot$ Arnoud de Kok $^{3}$ - Matthias Verstraete ${ }^{3}$ Tom Van Hoof ${ }^{3}$. \\ Catherine Van Der Straeten ${ }^{3} \cdot$ Mo Saffarini $^{4} \cdot$ Jan Victor $^{3}$
}

Published online: 24 July 2017

(C) European Society of Sports Traumatology, Knee Surgery, Arthroscopy (ESSKA) 2017

\section{Erratum to: Knee Surg Sports Traumatol \\ Arthrosc (2017) 25:1720-1730 \\ DOI 10.1007/s00167-016-4330-8}

Unfortunately, one of the author names was incorrect in the original online publication of this article. The correct name is given below:

Catherine Van Der Straeten.

The online version of the original article can be found under doi:10.1007/s00167-016-4330-8.

Michel P. Bonnin

bonnin.michel@gmail.com

1 Centre Orthopédique Santy, 24 Av Paul Santy, Lyon, France

2 Hopital Privé Jean Mermoz, 55 Av Jean Mermoz, 69008 Lyon, France

3 Department of Orthopaedics, Ghent University, De Pintelaan, 185, Ghent, Belgium

4 Accelerate Innovation Management, Rue de Hollande 4-6, 1204 Geneva, Switzerland 